\title{
Intravital microscopy identifies selectins that regulate $T$ cell traffic into allografts
}

\author{
Thomas R. Jones, Nozomu Shirasugi, Andrew B. Adams, Thomas C. Pearson, \\ and Christian P. Larsen \\ Emory Transplant Center and Department of Surgery, Emory University School of Medicine, Atlanta, Georgia, USA
}

$\mathrm{T}$ cell homing to sites of injury and inflammation is a critical step for adaptive immune responses. While much has been learned regarding $\mathrm{T}$ cell homing to lymphoid tissues, few studies have directly observed trafficking events during an effector response. In this study, we developed a model that uses intravital fluorescence videomicroscopy to determine the molecules critical to $T$ cell rolling within skin allograft microvasculature during the effector phase of the rejection response. Additional studies were performed to quantify $\mathrm{T}$ cell infiltrates as rejection progressed. We found that $\mathrm{P}$-selectin and E-selectin expressed on postcapillary venules play overlapping roles in the recruitment of activated $T$ cells in a SCID reconstitution model of skin graft rejection and are important in $T$ cell accumulation at the graft site. Surprisingly, we also found that naive $T$ cells are recruited and accumulate via constitutive $\mathrm{T}$ cell $\mathrm{L}$-selectin and upregulated $\mathrm{L}$-selectin ligands on rejecting allograft vasculature. These data indicated that a specific retinue of molecules is upregulated during the rejection response, and they suggest potential future therapeutic targets.

J. Clin. Invest. 112:1714-1723 (2003). doi:10.1172/JCI200319391.

\section{Introduction}

The successful generation of adaptive immune responses depends on leukocyte trafficking events. These events involve the movement of specialized populations of APCs and naive and effector lymphocytes to their sites of action in a coordinated fashion. DCs, acting as sentinels, transport antigens from peripheral sites of inflammation to secondary lymphoid organs for presentation to the adaptive immune system. To enhance the likelihood that rare antigen-specific $\mathrm{T}$ cells will encounter antigen-laden DCs, naive and subpopulations of memory $\mathrm{T}$ cells continuously recirculate through $\mathrm{LNs}$, entering via high endothelial venules. Once activated, antigen-specific $\mathrm{T}$ cells are mobilized into the bloodstream via efferent lymphatics and are ultimately recruited into peripheral sites where the eliciting antigen is expressed to perform their effector functions (1).

In the past decade, there have been major advances in our understanding of the molecular interactions that govern lymphocyte traffic. Much of this information has come from the study of the interactions of naive $T$ cells with high endothelial venules in peripheral LNs. In this setting, $\mathrm{T}$ cell recruitment involves a three-step

Received for publication July 2, 2003, and accepted in revised form October 14, 2003.

Address correspondence to: Christian P. Larsen, Emory Transplant Center and Department of Surgery, Suite 5105, Woodruff Memorial Building 1639, Pierce Drive, Atlanta, Georgia 30322, USA. Phone: (404) 727-8465; Fax: (404) 727-3660; E-mail: clarsen@emory.org.

Conflict of interest: The authors have declared that no conflict of interest exists.

Nonstandard abbreviations used: endothelial cell (EC); intravital fluorescence videomicroscopy (IVM); C57BL/6 (B6); high molecular weight (HMW). process during which the lymphocyte rolls along the endothelial surface, then firmly adheres, and ultimately traverses the vessel wall to the extravascular space. Low-strength selectin-molecule interactions mediate the rolling phase. This results in a reduction in velocity that allows the lymphocyte to sample the endothelial microenvironment for soluble chemokines that bind tightly regulated $\mathrm{G}$ protein-coupled receptors. Activation of these "serpentine" receptors triggers a rapid conformational change of surface integrin molecules on the lymphocyte. Integrin binding mediates arrest of the lymphocyte in close association with the endothelium, and subsequent signaling directs diapedesis of the cell through the endothelial layer into the tissue (2). Each of these interactions requires the expression of complementary receptors and ligands on the lymphocyte and endothelial cells (ECs). There are also exceptions to the general model of $T$ cell trafficking in which certain molecules possess the dual functions of rolling and firm adhesion, or adhesion and diapedesis (3).

The best-defined molecules that regulate rolling interactions are L-selectin, expressed on T cells, and E- and Pselectins, expressed on activated endothelium. In T cell trafficking to secondary lymphoid tissues such as LNs, the reduction in velocity mediated by L-selectin is critical for adhesion to and extravasation from the high endothelial venules (4). Similarly, enzymatic modification of P-selectin ligands, such as cutaneous leukocyte antigen in skin-homing $\mathrm{T}$ cells, upon activation is important for effector cell homing $(5,6)$. Deficiencies in the enzymes necessary for such modifications, such as fucosyltransferases, have been shown to inhibit peripheral homing of T cells $(7,8)$.

As with other responses, leukocyte trafficking events in transplantation begin with the migration of donor tissue DCs to draining LNs, where host $\mathrm{T}$ cells are acti- 
vated by recognition of foreign MHC in the direct alloresponsive pathway $(9,10)$. Alternatively, host DCs can acquire donor antigens and present them to recipient $T$ cells via the indirect allorecognition pathway. Activated $\mathrm{T}$ cells then enter the bloodstream, where ECs are the first cells within a vascularized allograft to interact with host immune cells.

Several studies have demonstrated roles for chemoattractant molecules in the process of graft rejection. While much has been learned about the role of selectins in ischemia/reperfusion injury, little is known about the necessity of these signals in lymphocyte homing to allografts (11-13). Knockout models have indicated a role for selectin molecules in lymphocyte recruitment, but with only modest impacts on graft survival (13-15). Inhibition or absence of chemokines or their receptors has yielded prolongations in heart allograft survival, with temporary reductions in the magnitude of the $\mathrm{T}$ cell infiltrate as determined by immunohistochemical staining of graft sections $(16,17)$. While such findings are promising, few studies have been able to directly identify homing as a mechanism for prolonged graft survival, given the overlapping costimulatory roles of many adhesion molecules. In particular, the molecules governing the earliest recruitment of effector $\mathrm{T}$ cells to tissue allografts have yet to be characterized, and none of the recruitment events has been directly observed in vivo.

Here, we have developed a mouse skin-transplantation model that uses intravital fluorescence videomicroscopy (IVM) to directly study the molecules that mediate rolling of $\mathrm{T}$ cells during the effector phase of the rejection response. This technique allows observation of short-term rolling and firm adhesion interactions (18). In order to determine how rolling interactions facilitate $\mathrm{T}$ cell accumulation within a graft, we also used indium111-labeled ( In $^{111}$-labeled) cells in a 16-hour trafficking assay $(19,20)$. We find that, as rejection progresses, both activated and naive $T$ cells become increasingly recruited through enhanced rolling and firm adhesion interactions. We find that naive cells are recruited to the graft site through L-selectin, and that inhibition of the L-selectin pathway eliminates rolling behavior, resulting in elimination of firm adhesion events and subsequent accumulation of naive cells in the graft. Similarly, we demonstrate that P-selectin glycoprotein ligand interactions with $\mathrm{P}$ - and E-selectin are necessary for in vivo-activated $\mathrm{T}$ cell rolling, firm adhesion, and accumulation.

\section{Methods}

Mice. Adult male 4- to 6-week-old C57BL/6 (B6) (H-2b), BALB/c (H-2 $\left.{ }^{\mathrm{d}}\right)$, and B6 SCID $\left(\mathrm{H}-2^{\mathrm{b}}\right)$ mice were purchased from The Jackson Laboratory (Bar Harbor, Maine, USA). Animals received humane care in accordance with institutional guidelines.

Skin grafting and adoptive transfer. Full-thickness ear skin grafts $\left(\sim 1 \mathrm{~cm}^{2}\right)$ were transplanted on the dorsal thorax of B6 SCID recipients and secured with a plastic adhesive bandage for 6 days. The grafts were allowed to heal for 1 month (21), at which time engrafted B6
SCID mice received $2 \times 10^{7}$ nylon wool-passed wildtype $\mathrm{B} 6$ splenocytes via the penile vein.

T cell preparations. All B6 T cells for intravital-observation or accumulation studies were isolated using an automated magnetic cell sorting (autoMACS) system with magnetic bead-conjugated anti-mouse CD90 (Thy1.2) antibody (all from Miltenyi Biotec Inc., Auburn, California, USA). In some experiments, purified T cells were activated in vitro using a protocol modified from that described by Weninger et al. (22). Briefly, T cells were placed at a 1:1 stimulator/responder ratio in culture medium with $10 \mathrm{ng} / \mathrm{ml}$ recombinant murine IL-2 (rIL-2; BD Biosciences/Pharmingen, San Diego, California, USA). Stimulators were irradiated BALB/c splenocytes. After 5 days, cells were retrieved and purified using an annexin-based Dead Cell Removal Kit (Miltenyi Biotec Inc.). Analysis of cells was carried out using fluorochrome-conjugated antibodies (BD Biosciences). Flow cytometry was performed using a FACSCalibur, and data were analyzed using CellQuest software (both from Becton Dickinson Immunocytometry Systems, San Jose, California, USA). In both naive-cell isolates and activated-cell cultures, purity of $\mathrm{T}$ cells was greater than $95 \%$ by flow cytometry. Naive status of purified cells was verified by flow cytometric analysis of the key surface markers CD62L and CD44 and by intracellular cytokine staining for IFN- $\gamma$ following stimulation with allogeneic targets. The $\mathrm{T}$ cells failed to produce IFN- $\gamma$ in response to allogeneic targets, as opposed to the vigorous response of $\mathrm{T}$ cells from mice that had been previously challenged with donor-specific grafts. Cells had a characteristic naive phenotype of CD62L high (>95\%) and CD44 low (>98\%) compared with $\mathrm{T}$ cells from donor-sensitized mice and activated T cells. In vitro-activated cells were characteristically CD62L low and CD44 high.

Antibodies. Blocking reagents included anti-CD62L antibody (purified from MEL-14 hybridoma; American Type Culture Collection, Manassas, Virginia, USA) or rat IgG2a isotype control (BD Biosciences); anti-CD62P or rat IgG1 (both from BD Biosciences); anti-CD62E or rat IgG2a (both from BD Biosciences); and CD62P-Ig fusion protein (BD Biosciences) or human IgG1 (Jackson ImmunoResearch Laboratories Inc., West Grove, Pennsylvania, USA).

CFSE labeling. Cells were labeled with the fluorescent dye CFSE. Purified naive or activated B6 T cells were incubated in $10 \mu \mathrm{M}$ CFSE (Molecular Probes Inc., Eugene, Oregon, USA). After 15 minutes, the staining was halted by the addition of cold RPMI 1640 with $10 \%$ FBS. Cells were then resuspended in sterile saline.

Preparation for intravital observations. At $0,1,3$, or 4 days after reconstitution with syngeneic T cells, B6 SCID recipients of skin grafts were anesthetized and placed on a Universal specimen holder (Carl Zeiss Inc., Thornwood, New York, USA) fitted with a $24 \mathrm{~mm} \times 40 \mathrm{~mm}$ coverslip (VWR, West Chester, Pennsylvania, USA). The descending aorta was cannulated via the left carotid artery using a PE-10 polyethylene catheter (Becton Dickinson). All animals received an infusion of high-molecular weight (HMW; $155 \mathrm{kDa}$ ) TRITC-conjugated dex- 
tran (Sigma-Aldrich, St. Louis, Missouri, USA) prior to observation to provide contrast to the microvasculature (see Supplemental Video 1 for an example of visualized flow; www.jci.org/cgi/content/full/112/11/1714/DC1). Intravital microscopy. SCID recipients of well-healed allografts were prepared as above and transferred to an intravital microscope (IX-70) fitted with infinity-corrected water-immersion objectives $(\times 10$ and $\times 20$, resulting in $\times 100$ and $\times 200$ magnification, respectively) and a dual-wavelength filter for detection of red and green fluorescence (all from Olympus America Inc., Melville, New York, USA). Boluses of CFSE-labeled naive or activated cells were infused into the carotid artery catheter. Cells that entered the skin graft microvasculature were visualized using fluorescent illumination. Images were visualized with a DXC390 CCD camera, displayed on a Trinitron PVM-14M2MDU monitor, and recorded using an SVHS VCR SVO9500MD (all from Sony Corp., Tokyo, Japan) for off-line analysis. A time generator accurate to 0.01 seconds was also used (FOR-A Corp., Cypress, California, USA). A stage micrometer (Olympus America Inc.) image was captured before each experiment for distance measurements. In some experiments, fluoresceinated antibodies were infused through the catheter. These were allowed to circulate for 20 minutes. The inferior vena cava was cut, and the animal was perfused with $4 \mathrm{ml}$ of saline to wash nonbinding antibody. The vasculature of the skin graft was analyzed for staining.

Analysis of intravital recordings. Rolling and adherent cells were defined as described in Vajkoczy et al. (3). Briefly, measurements of the skin microvascular included the diameter $\left(d_{v}\right)$ of the postcapillary venules, and the velocity $\left(V_{t}\right)$ and diameter $\left(d_{t}\right)$ of observed $T$ cells. The highest velocity of a noninteracting cell for a given venule $\left(V_{\max }\right)$ was used to calculate the mean blood flow velocity $\left(V_{\text {mean }}\right)$ as follows: $V_{\text {mean }}=V_{\max } /\left(2-\varepsilon^{2}\right)(\mu \mathrm{m} / \mathrm{s})$, where $\varepsilon$ is the ratio of the average $\mathrm{T}$ cell diameter $\left(d_{\mathrm{t}}\right)$ to the venule diameter $\left(d_{v}\right)$. Alternatively, $V_{\text {mean }}$ was determined as an average of noninteracting-cell velocities. Both methods yielded similar values and identical experimental results. Adherent cells were defined as those having a velocity of 0 for at least 20 seconds in a clearly patent vessel, i.e., no flow stasis as visualized by HMW TRITC-conjugated dextran. Rolling cells were identified by comparison of velocities to a critical threshold defined by the flow dynamics for a vessel with an idealized parabolic velocity profile: $V_{\text {crit }}=V_{\text {mean }} \times \varepsilon \times(2-\varepsilon)$. Cells traveling below the critical velocity were defined as rolling. The data presented herein are the mean percentage of total flux of $\mathrm{T}$ cells undergoing the specified interaction.

Quantitative PCR. Skin grafts were harvested before or at time points following reconstitution with $\mathrm{B} 6 \mathrm{~T}$ cells and snap-frozen in liquid nitrogen. RNA was isolated using TRIZOL reagent (Sigma-Aldrich), and cDNA was made using a first-strand cDNA synthesis kit (Invitrogen Corp., San Diego, California, USA). cDNA was then analyzed using the SYBR Green PCR kit (Applied Biosystems, Foster City, California, USA) with appropriate primers for testing of $\beta$-actin, CD62E, CD62L, and
CD62P. A GeneAmp 5700 PCR system (Perkin-Elmer, Wellesley, Massachusetts, USA) was used to amplify primed message and detect fluorescence incorporation of the SYBR Green reagent. The threshold cycle of fluorescence detection of the tested primer relative to that for actin in the sample of interest was compared with the results from the same primers in the control samples. The mathematical result is the fold change in expression of the sample of interest relative to controls.

In ${ }^{111}$ labeling and accumulation analysis. Naive or activated T cells were labeled with $20 \mu \mathrm{Ci} / 10^{7}$ cells $\operatorname{In}^{111}$ oxine $\left(t_{1 / 2}=2.8\right.$ days; Amersham Healthcare Atlanta, Atlanta, Georgia, USA) in RPMI $1640,10 \% \mathrm{FBS}$, at $37^{\circ} \mathrm{C}$ for 15 minutes. Cells were washed three times and then resuspended in sterile saline for injection into B6 SCID mice at day 0 or day 4 after reconstitution. Dilutions of each labeled cell population $\left(10^{5}, 5 \times 10^{4}, 2.5 \times 10^{4}, 1.25 \times 10^{4}, 6.25 \times 10^{3}\right.$, and $3.12 \times 10^{3}$ ) were retained to measure $\gamma$ reactivity for labeling efficiency. Tissue samples were harvested 16 hours after infusion, and $\gamma$ activity was measured. Cell numbers were calculated based on a linear regression computed from the signal/cell number ratio of the previous dilutions recalculated to account for decay.

Statistical analysis. Two-tailed Student's $t$ tests were used to determine statistical significance, defined as a $P$ value less than 0.05 .

\section{Results}

Tcell rolling and firm adhesion events increase during rejection. We first sought to develop a model in which to study the mechanisms involved in T cell-EC interactions and T cell recruitment in vivo during the early stages of allograft rejection. For this we transplanted dorsal ear skin from $\mathrm{BALB} / \mathrm{c}$ or $\mathrm{B} 6$ donors onto B6 SCID immunodeficient recipients. The grafts were observed for 1 month to allow time for revascularization. By this time a complex network of arterioles, capillaries, and venules could be observed by intravital microscopy. Intravenous injection of HMW TRITC-conjugated dextran, which is retained in the circulation, provided contrast to document revascularization (Supplemental Video 1, www.jci.org/cgi/content/full/112/11/1714/DC1).

To initiate rejection in this system, the skin graft recipients were reconstituted with naive wild-type B6 $\mathrm{T}$ cells. Under such circumstances the allografts were uniformly rejected between 11 and 14 days after $T$ cell reconstitution (median survival time $=12$ days $)(21$, 23). Syngeneic grafts showed no signs of immunologic injury and survived indefinitely. Given the readily observable graft vasculature and reproducible rejection kinetics, this model provided an ideal environment in which to study $\mathrm{T}$ cell-EC interactions and $\mathrm{T}$ cell recruitment during allograft rejection in vivo.

In our initial experiments to visualize EC-T cell interactions, we reconstituted B6 SCID mice with wild-type B6 T cells 1 month after transplantation of allogeneic BALB $/ \mathrm{c}\left(\mathrm{H}-2^{\mathrm{d}}\right)$ or syngeneic B6 (H-2 $)$ skin grafts. At 0,1 , 3,4 , or 5 days after reconstitution and initiation of the rejection response, the descending aorta was cannulated 


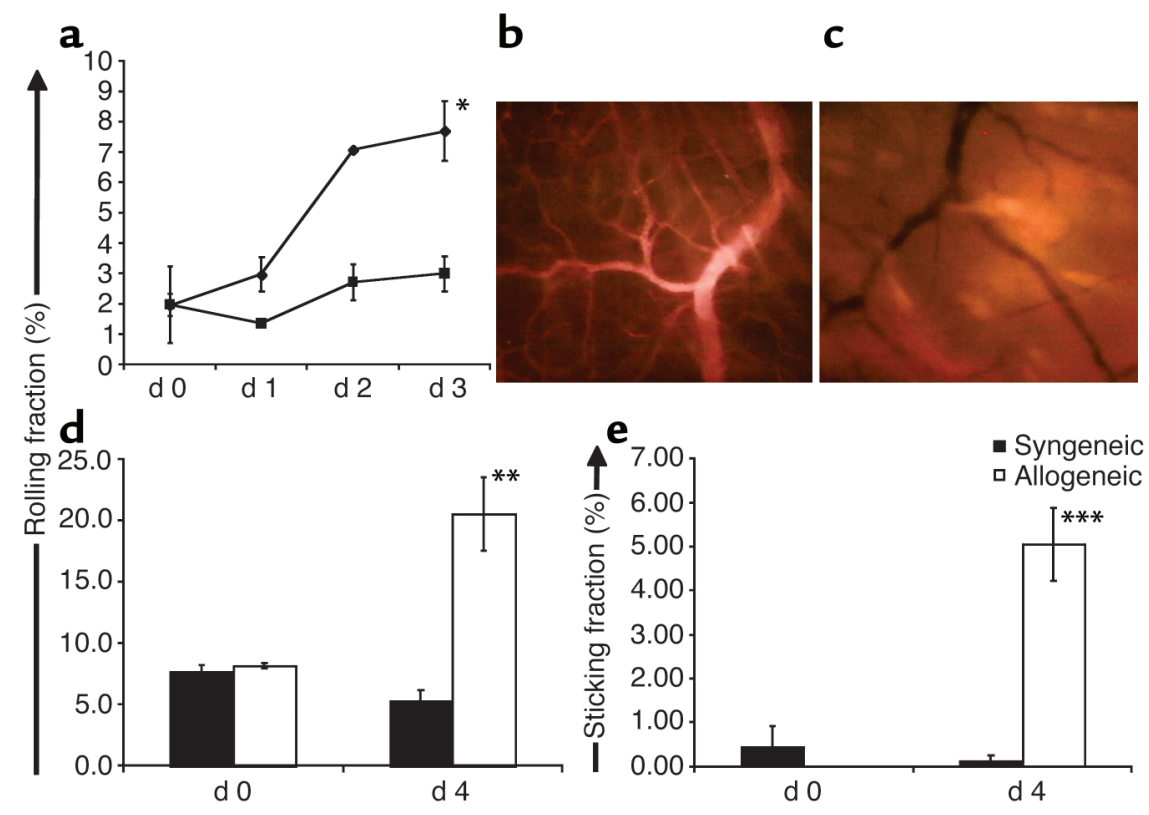

\section{Figure 1}

Graft rejection leads to enhanced rolling and firm adhesion interactions. (a) Adhesive interactions of CFSE-labeled naive T cells were analyzed at four time points after T cell reconstitution in venules of 1-month-healed syngeneic B6 (squares) and BALB/c allogeneic (diamonds) skin grafts on B6 SCID recipients. Rolling increased significantly in allografts as rejection progressed, and it remained equivalent at all time points in syngeneic grafts. ${ }^{*} P<0.04$. Data are mean \pm SEM, representative of three experiments; $n=3$ in each group. (b) At day 4 of rejection, all vessels clearly illuminated with HMW TRITC-conjugated dextran. (c) At day 5 of rejection, vascular damage and interrupted flow were evident, as many small vessels $(<50 \mu \mathrm{m}$ ) failed to illuminate with TRITC-dextran in allografts. ( $\mathbf{d}$ and $\mathbf{e})$ CFSE-labeled, activated T cells were infused at day 0 or day 4 after $T$ cell reconstitution in SCID recipients of syngrafts and allografts. Labeled cells demonstrated equivalent rolling $(\mathbf{d})$ and adhesion (e) at day 0 after reconstitution in BALB/c allografts (white bars) and B6 syngrafts (black bars) but showed a dramatic increase in rolling and firm adhesion by day 4 in allografts. ${ }^{*} P<0.04,{ }^{*}{ }^{*} P<0.03$. Data are mean \pm SEM; $n=3$ in each group.

via the left carotid artery, and CFSE-labeled naive T cells were infused to allow observation of T cell-EC interactions in vessels illuminated with TRITC-conjugated dextran. Animals were sacrificed at each time point; therefore, each data point represents the mean percentage of total cell flux interacting in separate animals $(n=3$ for each group). At day 0 , interactions with graft vasculature in BALB/c and B6 grafts were indistinguishable (mean rolling fraction $1.9 \%$ in allografts, $1.9 \%$ in syngrafts; Figure $1 \mathrm{a})$, but by days 3 and 4 of the rejection response, naive $T$ cells began to roll specifically in BALB/c allografts (mean rolling fraction $7.7 \%$ vs. $2.9 \%$ in syngeneic controls, $P<0.04$; Figure 1a). Firm adhesion events, which were nonexistent at early time points, occurred in less than $0.5 \%$ of total naive-cell flux by day 4 . Day 4 grafts consistently demonstrated uninterrupted vascular flow (Figure 1b; Supplemental Video 2a, www.jci.org/cgi/content/ full/112/11/1714/DC1). Interestingly, by day 5 , a significant portion of the graft microvasculature failed to illuminate with the dextran, and the remaining flow was sluggish or limited to larger vessels (Figure 1c; Supplemental Video $2 \mathrm{~b}$ ). As a result, the paucity of cells entering graft vasculature and the reduced visual clarity due to increasing graft edema at day 5 precluded analysis of interactions at or beyond this time point. Interestingly, the first macroscopic signs of graft rejection, such as discoloration and ulceration, did not appear until day 7 , and total graft loss did not occur until day 12 (21).
Next, we studied interactions between donor-specific activated $T$ cells and ECs in syngeneic and allogeneic skin grafts at day 0 and day 4 of rejection. Activated cells were generated in vitro as described in Methods. Cells from these cultures had an activated phenotype (CD44 high, CD62L low) and were greater than $93 \% \mathrm{CD}^{+} \mathrm{T}$ cells. A significant proportion of cells engaged in rolling behavior in both syngeneic and allogeneic grafts at day 0 (mean rolling fraction $7.7 \%$ and $8.2 \%$, respectively, $P=0.51$; Figure 1d); however, at day 4 of the rejection response, rolling interactions were significantly more frequent in allogeneic grafts, remaining unchanged in syngeneic grafts (mean rolling fraction $20.5 \%$ and $5.3 \%$, respectively, $P<0.04$; Figure 1d). Firm adhesion interactions, which were relatively rare at day 0 , were also increased in allogeneic grafts at day 4 of the rejection response (mean sticking fraction $5.5 \%$ vs. $0.13 \%$ in syngeneic grafts, $P<0.03$; Figure $1 \mathrm{e}$ ).

Intravital microscopy of skin grafts provides for observations of the short-term rolling and adhesion interactions but, due to technical limitations, does not allow long-term measurements of cellular accumulation over hours or days. Therefore, in order to determine how rolling and firm adhesion interactions translate into $\mathrm{T}$ cell accumulation in skin grafts at different time points during rejection, we labeled highly purified naive or activated T cells with $\operatorname{In}^{111}(19,20)$. These cells were given by intravenous injection to SCID recipients of both an allogeneic (left dorsal thorax) and a syn- 

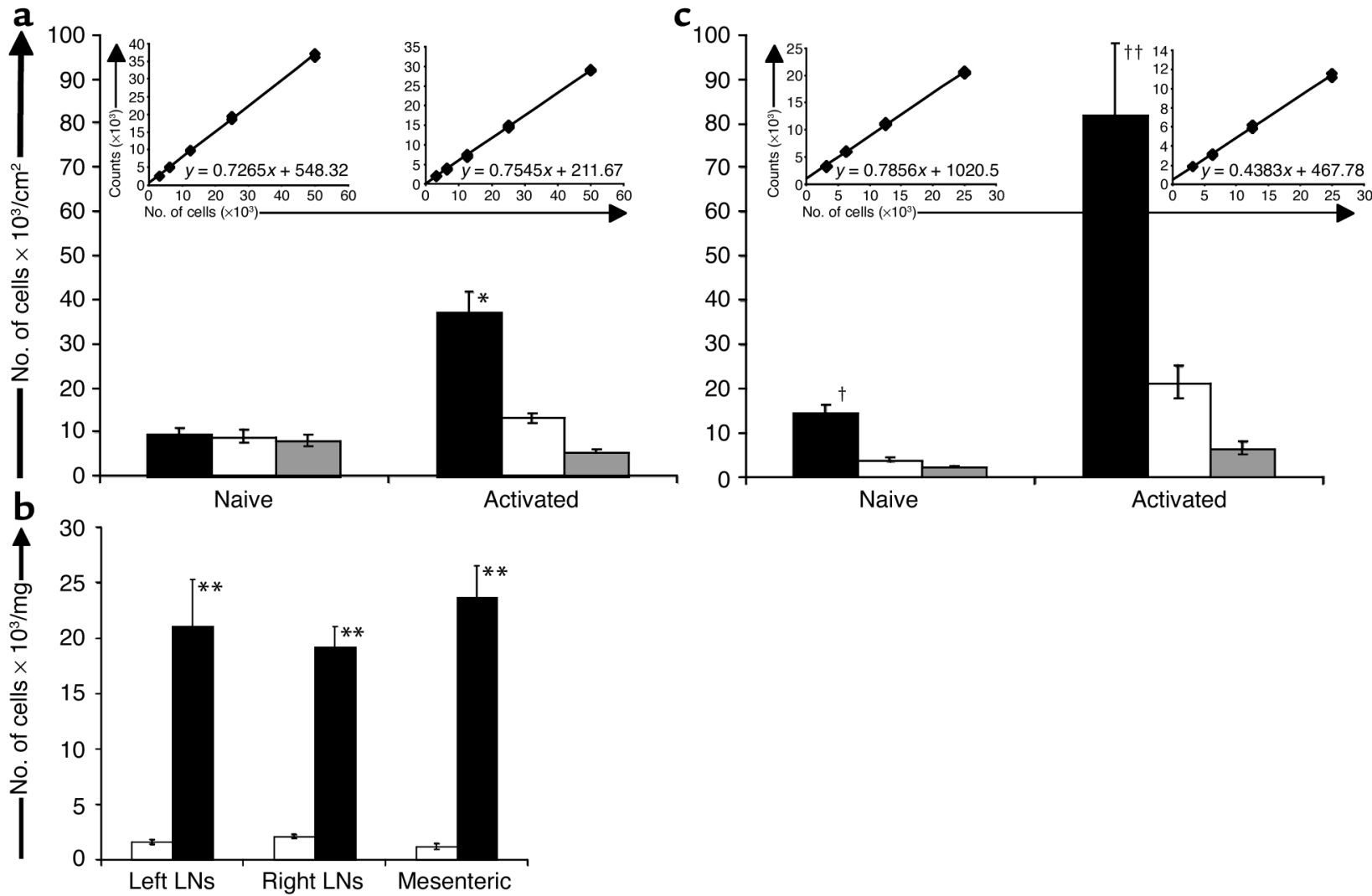

\section{Figure 2}

T cell accumulation in grafts corresponds to the increased rolling and firm adhesion interactions. Naive or activated T cells were labeled with $\mathrm{In}^{111}$. Cells were infused intravenously into B6 SCID recipients of both an allograft and a syngraft. Grafts and other tissues were harvested to record $\gamma$ activity. Insets show the linear regression accounting for decay of the signal recorded from known numbers of labeled cells for each experiment. (a) At day 0 after reconstitution, naive T cells accumulated equivalently in allografts (black bars), syngrafts (white bars), and normal ear (gray bars), whereas activated T cells accumulated to a significantly greater extent in allografts. ${ }^{*} P<0.002$ vs. syngrafts. Data are mean \pm SEM, representative of two experiments; $n=4$ in each group. (b) At day 0 after reconstitution, naive cells (black bars) were recruited to LNs ipsilateral (left LNs) and contralateral (right LNs) to the allograft, and to mesenteric nodes, with much greater affinity than were activated T cells (white bars). ${ }^{*} P<0.004$. Data are mean \pm SEM; $n=4$ in each group. (c) At day 4 post-reconstitution, both naive and activated $T$ cells were selectively recruited to rejecting allografts (black bars) compared with syngrafts (white bars) and normal ear (gray bars). ${ }^{\dagger} P<0.007,{ }^{\dagger \dagger} P<0.005$ vs. syngrafts. Data are mean $\pm \mathrm{SEM} ; n=4$ in each group.

geneic (right dorsal thorax) graft at day 0 or day 4 of the rejection response. Tissue samples, including grafts, spleen, LNs (axillary, inguinal, brachial, and mesenteric), liver, and ear skin (separated from underlying cartilage), were taken 16 hours after intravenous infusion, and the $\gamma$ signal was measured. The signal was compared with a linear regression of the signal/cell number ratio recorded after labeling, and the cell number in the tissue was calculated after accounting for radioactive decay. Cell numbers were normalized to tissue mass (in milligrams) for spleen, liver, and LNs and to tissue area (in square centimeters) for skin samples.

As expected, there was no difference in accumulation of naive $T$ cells at day 0 of rejection between allografts, syngrafts, and normal ear. Interestingly, activated T cells showed significantly enhanced accumulation in syngeneic grafts at day 0 compared with normal ear skin $\left(1.3 \times 10^{4}\right.$ vs. $5.6 \times 10^{3}$ cells $/ \mathrm{cm}^{2}, P<0.003$; Figure $\left.2 \mathrm{a}\right)$. These data are consistent with the findings of Anderson et al. that syngeneic skin grafts have some upregulated factors in an mRNA expression profile months after engraftment in immunodeficient hosts (23). Accumulation of activated $\mathrm{T}$ cells in $\mathrm{BALB} / \mathrm{c}$ allografts at day 0 was observed at still higher levels $\left(3.7 \times 10^{4} \mathrm{cells} / \mathrm{cm}^{2}\right.$, $P<0.002$ vs. syngeneic; Figure $2 \mathrm{a}$ ). We also found that naive $T$ cells preferentially migrated to secondary lymphoid tissues to a much greater degree than activated cells $(P<0.004$ in all groups; Figure $2 b)$, consistent with previous findings (24).

On the fourth day of the rejection response, accumulation data corresponded to the specific increase in rolling and firm adhesion events in both naive and activated $T$ cells as observed using IVM, with enhanced accumulation of both cell types. Naive T cell accumulation in allografts was significantly greater than in syngeneic grafts $\left(1.5 \times 10^{4}\right.$ and $4 \times 10^{3}$ cells $/ \mathrm{cm}^{2}$, respectively, $P<0.007)$. As on day 0 , activated cells accumulated in syngeneic grafts with greater frequency than in normal ear $\left(2.1 \times 10^{4}\right.$ and $6.6 \times 10^{3}$ cells $/ \mathrm{cm}^{2}$, respectively, $P<0.022)$; however, accumulation in rejecting 

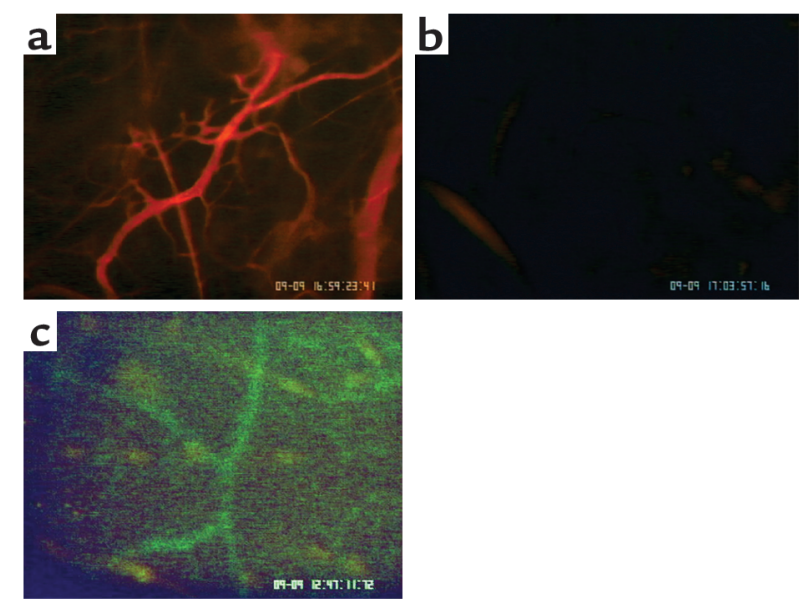

Figure 3

Intravital vascular staining of allografts. B6 SCID recipients of both an allograft and a syngraft were catheterized and received an injection of phycoerythrin-conjugated anti-mouse $\mathrm{K}^{\mathrm{d}}$ antibody at day 0 of rejection or FITC-conjugated anti-mouse CD62P at day 4 of rejection. After staining, the inferior vena cava was cut and $4 \mathrm{ml}$ of saline was washed through. Following $\mathrm{K}^{\mathrm{d}}$ staining, images were taken of graft vasculature in both the allograft (a) and the syngraft (b). $\mathrm{K}^{\mathrm{d}+}$ staining is clearly seen in allograft microvasculature and is restricted to vessels with a diameter of less than $50 \mu \mathrm{m}$. No staining was observed in syngrafts. Images are representative of five animals. (c) CD62P expression was only detectable at day 4 of rejection in skin allografts.

allografts was much greater than that in either syngeneic grafts or normal ear $\left(9 \times 10^{4}\right.$ cells $/ \mathrm{cm}^{2}, P<0.005$ vs. syngeneic, $P<0.001$ vs. normal ear; Figure $2 c$ ).

In addition, we wished to characterize the origin of the endothelium within the grafts. It is generally accepted that skin grafts are revascularized through invasion of the graft tissue by recipient-derived endothelium (25). Although it has been shown that donor factors play a role in angiogenesis, it is not known to what extent donor cells contribute to vascular endothelium (26). We therefore conducted intravital vascular labeling with anti-donor MHC class I antibody - specifically, phycoerythrin-conjugated anti-mouse $\mathrm{K}^{\mathrm{d}}$. BALB/c skin graft vasculature demonstrated significant staining with donor-specific antibody (Figure 3a), whereas B6 skin graft vessels failed to label (Figure $3 \mathrm{~b}$ ). The $\mathrm{K}^{\mathrm{d}+}$ vessels in the allografts were all less than $50 \mu \mathrm{m}$ in diameter, corresponding in size and structure to the postcapillary venules in which rolling and firm adhesion interactions had been observed in prior experiments, whereas the larger dermal vessels, previously shown to be recipient derived, failed to label (25).

To determine whether selectin expression was altered through the course of rejection, we performed intravital vascular staining to detect endothelial CD62P and $\mathrm{CD} 62 \mathrm{E}$ at different time points during rejection in allogeneic grafts and in syngeneic controls. Despite a predicted low-level constitutive expression of these molecules, we were unable to detect CD62P either in allografts at day 0 of rejection or in syngeneic grafts at any time point. However, CD62P staining was evident at day 4 of rejection (Figure 3c), but in neither syngeneic grafts nor grafts stained with isotype control.

Analysis of CD62E protein expression using this approach proved to be more difficult; therefore, we cannot draw conclusions regarding CD62E protein expression. Instead, we performed quantitative PCR analysis of skin grafts at day 4 of rejection relative to levels detected at day 0. While CD62P transcript levels were unchanged at day $4, \mathrm{CD} 62 \mathrm{E}$ transcripts were 2.5 -fold upregulated compared with those at day $0(P<0.03$ vs. syngeneic controls). These results are consistent with previous data that indicate that $\mathrm{CD} 62 \mathrm{P}$ expression is regulated post-transcriptionally through rapid release of preformed granules within ECs, while CD62E is regulated transcriptionally (27).

Naive T cell trafficking to rejecting skin allografts is dependent on CD62L interactions. Naive T cell trafficking to secondary lymphoid tissues has been well characterized, as the molecules governing the rolling and firm adhesion stages of lymphocyte recruitment to high endothelial venules are defined. $\mathrm{T}$ cell $\mathrm{L}$-selectin $(\mathrm{CD} 62 \mathrm{~L})$ is required for the rolling phase. Interruption of this pathway effectively prevents entry of naive cells into LNs and Peyer's patches and can significantly disrupt normal lymphocyte recirculation (4). As naive cells do not express the appropriate fucosyltransferase enzymes for modification of P- and E-selectin ligands on their surface that allow efficient binding, we hypothesized that the rolling behavior of naive $T$ cells at day 4 of rejection was CD62L dependent $(7,8)$.

As an initial approach to test this hypothesis, we revisited T cell trafficking in the IVM model. B6 SCID recipients of BALB/c allografts were reconstituted with naive B6 T cells 1 month after engraftment. Four days into the rejection response, recipients were catheterized and infused with CFSE-labeled naive or activated T cells in a suspension containing anti-mouse CD62L (MEL-14) or an isotype control. Interactions with graft vasculature were monitored. Naive $T$ cell rolling and adhesion events were essentially eliminated when CD62L was blocked, whereas interactions of control-treated $\mathrm{T}$ cells were comparable to previous results $(P<0.02$; Figure 4a; Supplemental Video 3a, www.jci.org/cgi/content/full/ $112 / 11 / 1714 / D C 1)$. In contrast, activated T cell-EC interactions were unaltered by anti-CD62L mAb's $(P=0.59$ vs. isotype controls; Figure 4b; Supplemental Video 3b).

Next we wished to determine whether interruption of the CD62L rolling pathway translated into diminished infiltration of the graft by naive $\mathrm{T}$ cells. We infused In $^{111}$-labeled naive T cells and anti-CD62L or isotype control into grafted $\mathrm{B} 6 \mathrm{SCID}$ recipients on day 4 of the rejection response. Tissues were collected 16 hours later. As previously observed in other models, antiCD62L treatment had a profound inhibitory effect on naive $T$ cell trafficking to LNs (Figure 4c) and increased the presence of cells in peripheral blood with no effect on trafficking to spleen (data not shown). We also found that anti-CD62L reduced accumulation of naive $\mathrm{T}$ cells in rejecting allografts to the level observed in 


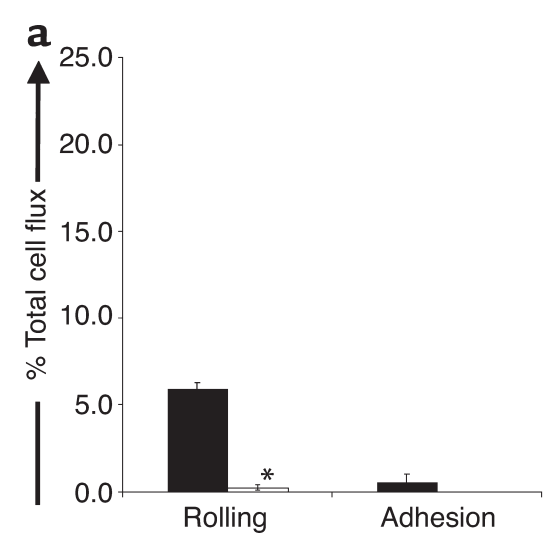

$\mathbf{b}_{25}$

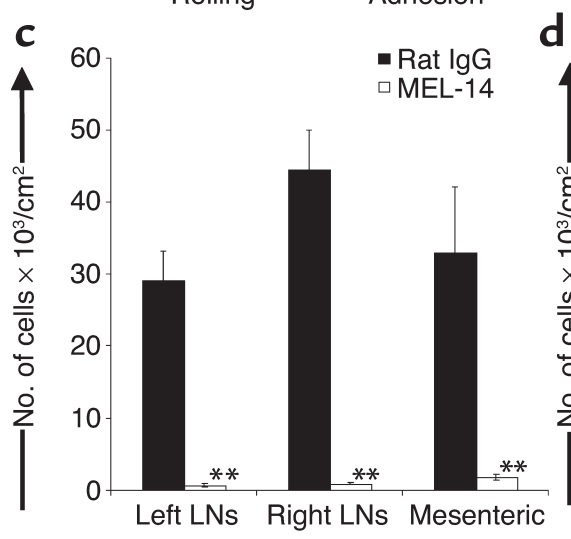

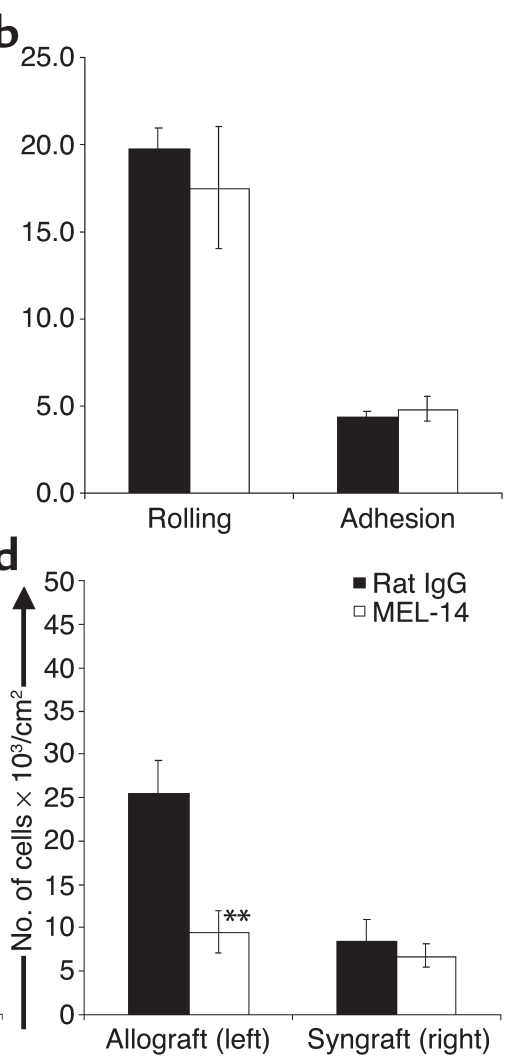

Figure 4

Rolling and accumulation of naive $T$ cells are dependent on CD62L interactions. CFSE- or $\ln ^{111}$. labeled naive or activated $T$ cells were infused with either anti-mouse CD62L or isotype control (rat $\operatorname{lgG} 2 \mathrm{a}$ ) for IVM experiments ( $\mathbf{a}$ and $\mathbf{b}$ ) or accumulation studies (c), in SCID recipients 4 days after reconstitution with wild-type B6 T cells. (a) Rolling interactions of naive $T$ cells were more than $96 \%$ eliminated in animals receiving anti$\mathrm{CD} 62 \mathrm{~L}$ treatment. Firm adhesion interactions were completely eliminated. ${ }^{*} P<0.02$. Data are mean $\pm \mathrm{SEM} ; n=3$ in each group. (b) Anti-CD62L treatment had no effect on the frequency of activated-cell rolling or firm adhesion events. (c) The control reveals that accumulation of naive T cells in the LNs was eliminated by treatment with MEL-14. (d) Like rolling and adhesion events, naive $T$ cell accumulation in allografts was significantly inhibited, reflecting the levels seen in syngrafts. ${ }^{*} P<0.02$ vs. isotype-treated controls. Data are mean \pm SEM; $n=4$ in each group. Each experiment was repeated with the same results. syngeneic grafts and normal ear $(P<0.02$; Figure $4 d)$. Together, these data indicate that naive $T$ cells require CD62L for the interactions with rejecting graft vascular endothelium, whereas activated $\mathrm{T}$ cells, which express low levels of CD62L, do not.

Trafficking of activated $T$ cells to rejecting skin allografts is dependent on distinct contributions from CD62P and CD62E. Activated $T$ cells demonstrated significant affinity for allograft endothelium in both IVM and accumulation experiments. Studies in TNF- $\alpha$-treated cremaster muscle and uninflamed skin have implicated CD62P and CD62E expression on the endothelium as important mediators of activated T cell-EC interactions in the periphery $(7,28,29)$. Both of these selectins display regulated expression. P-selectin is retained in granules within ECs that are released through inflammatory stimulation, while E-selectin is transcriptionally regulated. Based on previous studies of effector cell trafficking, either one of these molecules, both, or neither could play important roles in rolling, firm adhesion, and accumulation. Leukocytes interact with E- and P-selectins via both shared and specific ligands, the affinities of which depend on specific glycoprotein modifications.

We first designed an experiment to block selectin ligands borne by activated T cells. CFSE-labeled naive or activated $\mathrm{T}$ cells in $150 \mu \mathrm{g}$ of P-selectin-Ig fusion protein or isotype control were infused into catheterized graft recipients on day 4 of the rejection response. This fusion protein blocks selectin ligands that bind both $\mathrm{E}$ and P-selectins $(6,30)$. We found that CD62P-Ig had no effect on naive $\mathrm{T}$ cell rolling and firm adhesion $(P=0.55$ vs. isotype control; Figure 5a). In contrast, we found that the fusion protein profoundly inhibited the rolling of activated T cells ( $88 \%$ inhibition, $P<0.015$ vs. isotype control) and entirely eliminated activated $T$ cell firm adhesion (Figure 5b). As with CD62L blockade of naive cells, accumulation of In $^{111}$-labeled activated T cells was dramatically reduced $(60 \%, P<0.015)$ in animals receiving P-selectin-Ig (Figure 5c).

Finally, we wished to resolve the relative roles of CD62E and CD62P in rolling and firm adhesion. For this, we performed experiments on day 4 of the rejection response in which we observed the rolling behavior of activated T cells while blocking E-selectin, P-selectin, or both. In the first experiment, recipients received an infusion of anti-CD62P or isotype control. This was followed by four infusions of CFSE-labeled activated $\mathrm{T}$ cells for IVM analysis. The same animals then received another infusion of anti-CD62P with anti-CD62E or isotype controls, followed by four additional cell infusions. In another experiment, these treatments were reversed, with anti-CD62E given alone, followed by anti-CD62P with anti-CD62E. Whenever possible, the same vessels observed for the first set of infusions were reassessed for the second set. Blockade of CD62P alone resulted in a dramatic reduction in the frequency of rolling interactions (mean rolling fraction $4.4 \%$ vs. $22.5 \%$ in isotype controls, $P<0.002$; Figure 6a); however, only $50 \%$ of firm adhesion interactions were inhibited (mean sticking fraction $3.38 \%$ vs. $6.74 \%$ in isotype controls, $P<0.04$; Figure 6b; Supplemental Video 4a, www.jci.org/cgi/content/full/112/11/1714/DC1). The addition of anti- 

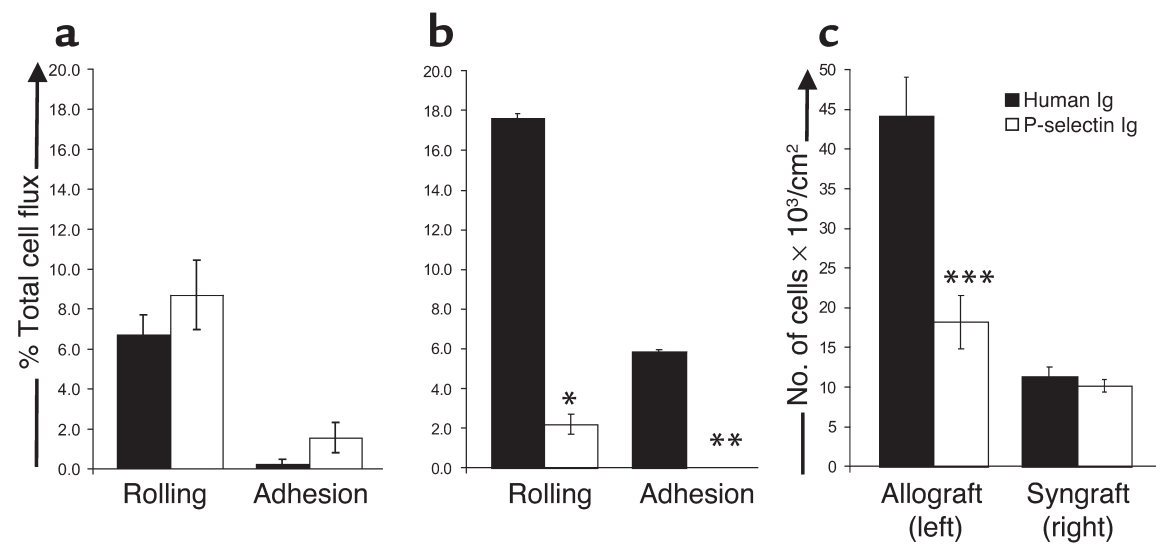

Figure 5

Blockade with CD62P-Ig fusion protein inhibits activated-cell rolling, adhesion, and accumulation. CFSE- or $\ln ^{111}$-labeled naive or activated $\mathrm{T}$ cells were infused in either CD62P-Ig fusion protein or human IgG1 for IVM experiments (a and $\mathbf{b})$ or accumulation studies (c) in SCID recipients 4 days after reconstitution with wild-type B6 T cells. (a) CD62P-Ig had no effect on naive T cell rolling or firm adhesion. (b) Activated cells treated with P-selectin-Ig fusion protein showed dramatically less rolling behavior than controls. Firm adhesion interactions were completely eliminated. ${ }^{*} P<0.015,{ }^{*} P<0.009$. Data are mean $\pm \mathrm{SEM} ; n=3$ in each group. (c) CD62P-Ig significantly inhibited activated T cell accumulation in allografts. ${ }^{* *} P<0.015 \mathrm{vs}$. isotype controls. Data are mean \pm SEM; $n=4$ in each group. Each experiment was repeated with the same results.

CD62E had no significant additional impact on rolling but eliminated most of the observed firm adhesion (mean sticking fraction $0.29 \%, P<0.007$ vs. isotype, $P<0.03$ vs. anti-CD62P alone; Figure 6b; Supplemental Video 4, b compared with c). In the reverse experiment, anti-CD62E again had little impact on the rolling interaction $(22.1 \%$ vs. $25.3 \%$ in isotype controls, $P=0.53$; Figure $6 c$ ) but significantly inhibited firm adhesion (3.9\% total flux vs. $6.84 \%$ in isotype controls, $P<0.02$; Figure $6 \mathrm{~d}$ ), while the addition of anti-CD62P eliminated the remaining rolling and firm adhesion interactions (mean rolling fraction $4.6 \%, P<0.02$ vs. anti-CD62E alone; mean sticking fraction $0.67 \%, P<0.008$ vs. anti-CD62E alone; Figure $6 \mathrm{~d}$ ). We can therefore conclude that, taken with the P-selectin-Ig data, these results indicate overlapping functions of endothelial CD62P and CD62E that rely on receptors borne on the $\mathrm{T}$ cells.

\section{Discussion}

Over the past several years, intravital microscopy has allowed the validation of the three-step model of leukocyte recruitment in vivo. Initially, studies using this technique focused on the recruitment of naive lymphocytes into secondary lymphoid organs, confirming the roll of selectins, chemokines, and integrins in the processes of rolling and adhesion to high endothelial venules (2). Much less is known about the molecular interactions involved in the recruitment of naive and activated lymphocytes into extralymphoid sites during immune responses, particularly allograft rejection. In this report, we have described a new model system using intravital microscopy coupled with indium-labeling techniques to study T cell-EC interactions and lymphocyte recruitment during rejection.
The classic three-step model of leukocyte trafficking has provided a useful conceptual framework to guide the study of the recruitment of cells to lymphoid and nonlymphoid tissues. Recent reports have revealed the critical importance of initial rolling interactions in both homeostatic leukocyte trafficking and recruitment during effector responses. Rolling involves the interactions of molecules and receptors that act both in concert and independently. In some models, rolling interactions can be divided into two separate but linked events, where one molecule facilitates an initial tethering event that reduces cell velocity, and a subsequent molecular interaction further reduces rolling velocity and transitions the cell to firm adhesion if the appropriate chemotactic signals are detected. For instance, in murine experimental autoimmune encephalitis models, P-selectin and VCAM- 1 mediate overlapping rolling and adhesion events, whereas, in other inflammatory models such as TNF- $\alpha$-treated cremaster muscle, P-selectin and E-selectin have been implicated in both rolling and adhesion $(29,31,32)$. In a study of constitutive leukocyte adhesion to quiescent skin, CD62P-dependent events initiated rolling, and CD62E bridged rolling and adhesion $(7,8)$. These studies reveal that one cannot predict which molecules are critical to rolling in a given effector response.

In our experiments, adapted from the dorsal skin model first described by Yamada et al. (33), both activated and naive $T$ cells were recruited into allografts during rejection, but through distinct pathways unique to their respective surface-molecule profiles. Both populations exhibited increasing levels of rolling and firm adhesion interactions with the graft endothelium as rejection progressed. These interactions translated into accumulation in the $\mathrm{In}^{111}$ cell trafficking model that was proportionate to the frequency of the rolling and adhesion interactions.

Consistent with earlier studies, we observed that the recruitment of activated $T$ cells during rejection occurred earlier and at higher levels than recruitment of their naive counterparts (20). Both rolling and firm adhesion events were also clearly more frequent for activated $\mathrm{T}$ cells than for naive $T$ cells. Using intravital microscopy, we were able to directly study the molecules necessary for rolling in this setting. Consistent with reports from nontransplantation models, activated $\mathrm{T}$ cells used CD62E and CD62P, but not $\mathrm{CD} 62 \mathrm{~L}$, to mediate rolling on vascular endothelium. Furthermore, our data indicated that activated $\mathrm{T}$ cells demonstrated a two-step rolling interaction, with 

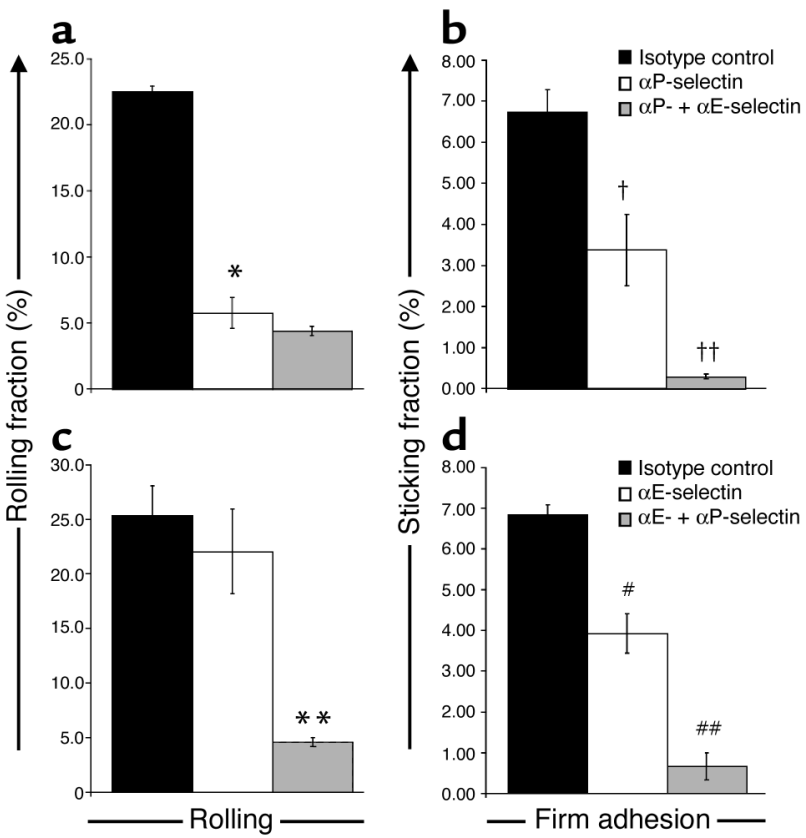

Figure 6

CD62P and CD62E play overlapping roles in rolling and firm adhesion. CFSE-labeled activated T cells were infused in $\mathrm{B} 6 \mathrm{SCID}$ recipients of $\mathrm{BALB} / \mathrm{c}$ allografts 4 days after reconstitution with wild-type B6 T cells for IVM experiments. Animals received the indicated treatments. (a) Rolling interactions were significantly inhibited by anti-mouse CD62P (white bars) versus isotype control (black bars). Additional antiCD62E with anti-CD62P (gray bars) did not significantly reduce rolling from that seen with anti-CD62P alone. ${ }^{*} P<0.002$ vs. isotype controls. Data are mean \pm SEM; $n=3$ in each group. (b) Firm adhesion events were significantly inhibited by anti-CD62P alone. Additional blockade with anti-CD62E further reduced these interactions. ${ }^{\dagger} P<0.04$ vs. isotype, ${ }^{\dagger} P<0.03$ vs. anti-CD62P alone and $P<0.007$ vs. isotype controls. Data are mean $\pm \mathrm{SEM} ; n=3$ in each group. (c) Rolling interactions were not affected by anti-CD62E treatment alone (white bars), compared to isotype control (black bars). Additional anti-CD62P (gray bars) reduced rolling to the levels observed in $\mathbf{a} .{ }^{*} P<0.02 \mathrm{vs}$. antiCD62E alone. Data are mean \pm SEM; $n=3$ in each group. (d) Anti$\mathrm{CD} 62 \mathrm{E}$ alone significantly inhibited firm adhesion interactions relative to isotype controls. Additional anti-CD62P further reduced these interactions. ${ }^{P} P<0.02$ vs. isotype control, $\# P<0.008$ vs. anti-CD62E alone. Data are mean $\pm \mathrm{SEM} ; n=3$ in each group.

endothelial CD62P mediating the initial tethering event, and $\mathrm{CD} 62 \mathrm{E}$ providing a causeway to firm adhesion. Blockade of the CD62P, but not the CD62E, pathway greatly diminished the incidence of activated $\mathrm{T}$ cell rolling in graft vasculature. However, a large fraction of the remaining rollers also firmly adhered to the endothelium. Additional blockade of CD62E eliminated the residual adhesion events. These data indicate that activated $\mathrm{T}$ cells use these two interacting pathways to enter allografts. Blockade with CD62P-Ig, which binds ligands that have affinity for both CD62E and CD62P, virtually eliminated rolling and firm adhesion of activated $\mathrm{T}$ cells in allografts. This translated into near complete elimination of accumulation of activated $\mathrm{T}$ cells, indicating that these rolling and firm adhesion interactions play a nonredundant role in the recruitment process.
These observations are consistent with those of Yamada et al., in which P-selectin-deficient animals demonstrated a reduction, but not elimination, of basal leukocyte rolling events in the dorsal skin chamber (33). The subsequent observations of Ley and colleagues in a similar model using E-selectin-deficient mice or an anti$\mathrm{CD} 62 \mathrm{E} \mathrm{mAb}$ demonstrated little impairment in the occurrence of rolling, but significant attenuation of adhesion events linked to increased rolling velocity (29). Other studies have since identified a cooperative function of P-selectin and E-selectin, where P-selectin mediates the initial tethering event and subsequent, highvelocity rolling, while E-selectin facilitates slow rolling that allows close sampling of soluble chemokines within the endothelial glycocalyx $(7,32)$. Each of these studies relied on observations of circulating leukocytes. Our findings build on these observations through the use of highly purified effector $\mathrm{T}$ cells within their target tissue during the effector response. While the endothelial selectins are generally considered to be initiators of immune responses in the periphery, our data support a role for these molecules in the expansion and amplification of the response after a small number of activated cells enter the graft. The vascular endothelium of both syngeneic and allogeneic skin grafts supports rolling and accumulation of activated cells at day 0 of rejection, and activated cells show an ability to specifically accumulate in allografts at this time point. These data support a model in which early recruitment of a small population of effector cells apparently leads to increased endothelial expression of CD62P and transcriptional upregulation of CD62E. These in turn mediate increased rolling, adhesion, and accumulation at later time points to facilitate rapid destruction of the graft vasculature.

Naive $T$ cells were not attracted to the graft at early time points, but later during the rejection response they showed increased rolling and accumulation. In contrast to the activated population, CD62L interactions accounted for essentially all rolling interactions and accumulation of naive $\mathrm{T}$ cells. These data suggest that while both CD62E/P- and CD62L-dependent mechanisms are invoked during the effector phase of allograft rejection, the expression of ligands for $\mathrm{CD} 62 \mathrm{~L}$ is upregulated with somewhat delayed kinetics compared with the expression of ligands for CD62P and CD62E. This recruitment of naive cells is somewhat surprising. Naive $\mathrm{T}$ cells are normally excluded from nonlymphoid tissues under homeostatic conditions and in the setting of acute inflammation (34). In the setting of chronic inflammation, such as autoimmunity, naive $T$ cells are recruited into peripheral sites. It is thought that this recruitment is dependent on the development of high endothelial venule-like structures in a process termed lymphoid neogenesis $(34,35)$. In the setting of skin allograft rejection, we have observed that naive $T$ cells are recruited to the site within 3-4 days of the initiation of the response. Thus it appears that there may be unique features of graft rejection that promote a more rapid recruitment of naive cells than has been observed in 
other disease models. Because of the high precursor frequency of alloreactive cells, some may be activated in the proinflammatory environment and thereby further amplify the immune response to the graft.

Our finding that the microvasculature of these skin grafts is a mosaic of donor- and recipient-derived ECs suggested that the recruiting endothelium is potentially an antigen-specific target of the immune response $(25,36)$. Consistent with this, we found that the graft endothelium undergoes severe damage that limits vascular flow 1 day after the most significant recruitment events occur. This change in hemodynamics precedes overt signs of rejection by another 1-2 days, raising the possibility that graft rejection in our model is in part due to tissue necrosis from lack of blood flow.

These data provide clues for the clinical application of trafficking-inhibitory reagents. In particular, blockade of trafficking events may help to prevent or treat episodes of acute rejection, providing more specific tools as potential alternatives to the regimens employed now, such as $\mathrm{T}$ cell depletion and high-dose steroid therapy. The presence of multiple synergistic pathways in this first step of $T$ cell trafficking to allografts suggests that it will be necessary to inhibit multiple targets in order to minimize allograft damage mediated by infiltrating lymphocytes. This may help to explain why transplantation studies in which a single rolling pathway has been interrupted have shown little if any survival advantage (15). It is important to develop a more complete understanding of the molecules that effector cells use to enter a graft. Here, we have established a framework for addressing these questions and have made an important first step by using a powerful new technology to visualize the rejection process in situ and thus gain insight into the molecular interactions that govern cell traffic into allografts.

\section{Acknowledgments}

We would like to thank Ulrich von Andrian for helpful discussions regarding this work. This research was supported by grants from the NIH and from the Carlos and Marguerite Mason Trust.

1. Banchereau, J., and Steinman, R.M. 1998. Dendritic cells and the control of immunity. Nature. 392:245-252.

2. Butcher, E.C., and Picker, L.J. 1996. Lymphocyte homing and homeostasis. Science. 272:60-66.

3. Vajkoczy, P., Laschinger, M., and Engelhardt, B. 2001. $\alpha 4$-IntegrinVCAM-1 binding mediates $G$ protein-independent capture of encephalitogenic T cell blasts to CNS white matter microvessels. J. Clin. Invest. 108:557-565. doi:10.1172/JCI200112440.

4. Warnock, R.A., Askari, S., Butcher, E.C., and von Andrian, U.H. 1998. Molecular mechanisms of lymphocyte homing to peripheral lymph nodes. J. Exp. Med. 187:205-216.

5. Robert, C., and Kupper, T.S. 1999. Inflammatory skin diseases, T cells, and immune surveillance. N. Engl. J. Med. 341:1817-1828.

6. Fuhlbrigge, R.C., Kieffer, J.D., Armerding, D., and Kupper, T.S. 1997. Cutaneous lymphocyte antigen is a specialized form of PSGL-1 expressed on skin-homing T cells. Nature. 389:978-981.

7. Weninger, W., et al. 2000. Specialized contributions by alpha(1,3)-fucosyltransferase-IV and FucT-VII during leukocyte rolling in dermal microvessels. Immunity. 12:665-676.

8. Homeister, J.W., et al. 2001. The alpha(1,3)fucosyltransferases FucT-IV and FucT-VII exert collaborative control over selectin-dependent leukocyte recruitment and lymphocyte homing. Immunity. 15:115-126.

9. Auchincloss, H., Jr., and Sultan, H. 1996. Antigen processing and presentation in transplantation. Curr. Opin. Immunol. 8:681-687.

10. Gould, D.S., and Auchincloss, H., Jr. 1999. Direct and indirect recognition: the role of MHC antigens in graft rejection. Immunol. Today. 20:77-82.

11. Fuller, T.F., et al. 2001. Reduction of severe ischemia/reperfusion injury in rat kidney grafts by a soluble P-selectin glycoprotein ligand. Transplantation. 72:216-222.

12. Farmer, D.G., et al. 2002. Improved survival through the reduction of ischemia-reperfusion injury after rat intestinal transplantation using selective P-selectin blockade with P-selectin glycoprotein ligand-Ig. Transplant. Proc. 34:985.

13. Tang, M.L., Hale, L.P., Steeber, D.A., and Tedder, T.F. 1997. L-selectin is involved in lymphocyte migration to sites of inflammation in the skin: delayed rejection of allografts in L-selectin-deficient mice. J. Immunol. 158:5191-5199.

14. Lacha, J., et al. 2002. Intercellular cell adhesion molecule-1 and selectin ligands in acute cardiac allograft rejection: a study on gene-deficient mouse models. J. Leukoc. Biol. 71:311-318.

15. Raisky, O., et al. 2001. Acute rejection and cardiac graft vasculopathy in the absence of donor-derived ICAM-1 or P-selectin. J. Heart Lung Transplant. 20:340-349.

16. Hancock, W.W., et al. 2000. Requirement of the chemokine receptor CXCR3 for acute allograft rejection. J. Exp. Med. 192:1515-1520.

17. Hancock, W.W., et al. 2001. Donor-derived IP-10 initiates development of acute allograft rejection. J. Exp. Med. 193:975-980.

18. von Andrian, U.H., and M'Rini, C. 1998. In situ analysis of lymphocyte migration to lymph nodes. Cell Adhes. Commun. 6:85-96.

19. Kupiec-Weglinski, J.W., Austyn, J.M., and Morris, P.J. 1988. Migration patterns of dendritic cells in the mouse. Traffic from the blood, and $\mathrm{T}$ cell-dependent and -independent entry to lymphoid tissues. J. Exp. Med. 167:632-645.

20. Larsen, C.P., Barker, H., Morris, P.J., and Austyn, J.M. 1990. Failure of mature dendritic cells of the host to migrate from the blood into cardiac or skin allografts. Transplantation. 50:294-301.

21. Bingaman, A.W., et al. 2000. Vigorous allograft rejection in the absence of danger. J. Immunol. 164:3065-3071.

22. Weninger, W., Crowley, M.A., Manjunath, N., and von Andrian, U.H. 2001. Migratory properties of naive, effector, and memory CD8(+) T cells. J. Exp. Med. 194:953-966.

23. Anderson, C.C., et al. 2001. Testing time-, ignorance-, and danger-based models of tolerance. J. Immunol. 166:3663-3671.

24. Masopust, D., Vezys, V., Marzo, A.L., and Lefrancois, L. 2001. Preferential localization of effector memory cells in nonlymphoid tissue. Science. 291:2413-2417.

25. Valujskikh, A., Lantz, O., Celli, S., Matzinger, P., and Heeger, P.S. 2002 Cross-primed CD8 $(+) \mathrm{T}$ cells mediate graft rejection via a distinct effector pathway. Nat. Immunol. 3:844-851.

26. Clark, R.A., et al. 1982. Fibronectin is produced by blood vessels in response to injury. J. Exp. Med. 156:646-651.

27. Kansas, G.S. 1996. Selectins and their ligands: current concepts and controversies. Blood. 88:3259-3287.

28. Walter, U.M., and Issekutz, A.C. 1997. Role of E- and P-selectin in migration of monocytes and polymorphonuclear leucocytes to cytokine and chemoattractant-induced cutaneous inflammation in the rat. Immunology. 92:290-299.

29. Ley, K., Allietta, M., Bullard, D.C., and Morgan, S. 1998. Importance of E-selectin for firm leukocyte adhesion in vivo. Circ. Res. 83:287-294.

30. Lenter, M., Levinovitz, A., Isenmann, S., and Vestweber, D. 1994. Monospecific and common glycoprotein ligands for E- and P-selectin on myeloid cells. J. Cell Biol. 125:471-481.

31. Kerfoot, S.M., and Kubes, P. 2002. Overlapping roles of P-selectin and alpha 4 integrin to recruit leukocytes to the central nervous system in experimental autoimmune encephalomyelitis. J. Immunol. 169:1000-1006.

32. Hickey, M.J., et al. 1999. Varying roles of E-selectin and P-selectin in different microvascular beds in response to antigen. J. Immunol. 162:1137-1143

33. Yamada, S., et al. 1995. Rolling in P-selectin-deficient mice is reduced but not eliminated in the dorsal skin. Blood. 86:3487-3492.

34. Weninger, W., et al. 2003. Naive T cell recruitment to nonlymphoid tissues: a role for endothelium-expressed CC chemokine ligand 21 in autoimmune disease and lymphoid neogenesis. J. Immunol. 170:4638-4648.

35. Takemura, S., et al. 2001. Lymphoid neogenesis in rheumatoid synovitis. J. Immunol. 167:1072-1080.

36. Pober, J.S., Orosz, C.G., Rose, M.L., and Savage, C.O. 1996. Can graft endothelial cells initiate a host anti-graft immune response? Transplantation. 61:343-349. 
